\title{
On Riemannian foliations admitting transversal conformal fields
}

\author{
Woo Cheol Kim and Seoung Dal Jung \\ (Received December 22, 2018) \\ (Revised October 21, 2019)
}

\begin{abstract}
Let $\left(M, g_{M}, \mathscr{F}\right)$ be a closed, connected Riemannian manifold with a Riemannian foliation $\mathscr{F}$ of nonzero constant transversal scalar curvature. When $M$ admits a transversal nonisometric conformal field, we find some generalized conditions that $\mathscr{F}$ is transversally isometric to $\left(S^{q}(1 / c), G\right)$, where $G$ is the discrete subgroup of $O(q)$ acting by isometries on the last $q$ coordinates of the sphere $S^{q}(1 / c)$ of radius $1 / c$.
\end{abstract}

\section{Introduction}

A Riemannian foliation is a foliation $\mathscr{F}$ on a smooth manifold $M$ such that the normal bundle $Q=T M / T \mathscr{F}$ may be endowed with a metric $g_{Q}$ whose Lie derivative is zero along leaf directions [15]. Note that we can choose a Riemannian metric $g_{M}$ on $M$ such that $\left.g_{M}\right|_{T_{\mathscr{F}}^{\perp}}=g_{Q}$; such a metric is called bundle-like. A Riemannian foliation $\mathscr{F}$ is transversally isometric to $(W, G)$, where $G$ is a discrete group acting by isometries on a Riemannian manifold $\left(W, g_{W}\right)$, if there exists a homeomorphism $\eta: W / G \rightarrow M / \mathscr{F}$ that is locally covered by isometries [10]. Recently, S. D. Jung and K. Richardson [6] proved the generalized Obata theorem which states that: $\mathscr{F}$ is transversally isometric to $\left(S^{q}(1 / c), G\right)$, where $G$ is the discrete subgroup of $O(q)$ acting by isometries on the last $q$ coordinates of the sphere $S^{q}(1 / c)$ of radius $1 / c$ if and only if there exists a non-constant basic function $f$ such that

$$
\nabla_{X} \nabla f=-c^{2} f X
$$

for all foliated normal vectors $X$, where $c$ is a positive real number and $\nabla$ is the transverse Levi-Civita connection on the normal bundle $Q$.

A transversal conformal field is a normal vector field with a flow preserving the conformal class of the transverse metric. That is, the infinitesimal auto-

This paper was also supported by the National Research Foundation of Korea (NRF) grant funded by the Korea government (MSIP) (NRF-2018R1A2B2002046).

2010 Mathematics Subject Classification. 53C12; 57R30.

Key words and phrases. Riemannian foliation, Transversal conformal field, generalized Obata theorem. 
morphism $Y$ is transversal conformal if $L_{Y} g_{Q}=2 f_{Y} g_{Q}$ for a basic function $f_{Y}$ depending on $Y$, where $L_{Y}$ is the Lie derivative. In this case, it is trivial that

$$
f_{Y}=\frac{1}{q} \operatorname{div}_{\nabla}(\pi(Y))
$$

where $\operatorname{div}_{\nabla}$ is a transversal divergence and $\pi: T M \rightarrow Q$ is the natural projection. If the transversal conformal field $Y$ satisfies $\operatorname{div}_{\nabla}(\pi(Y))=0$, i.e, $L_{Y} g_{Q}=0$, then $Y$ is said to be transversal Killing field, that is, its flow is a transversal infinitesimal isometry. The properties of the infinitesimal automorphisms have been studied by many authors ([4], [8], [13], [14], [16]).

In this article, we study the Riemannian foliation admitting a transversal nonisometric conformal field. First, we recall the well-known theorems about the Riemannian foliations admitting a transversal nonisometric conformal field ([3], [4], [5], [6], [12]).

Let $R^{Q}$, Ric $^{Q}$ and $\sigma^{Q}$ be the transversal curvature tensor, transversal Ricci operator and transversal scalar curvature with respect to the transversal LeviCivita connection $\nabla$ on $Q$ [15]. Let $\kappa_{B}$ be the basic part of the mean curvature form $\kappa$ of the foliation $\mathscr{F}$ and $\kappa_{B}^{\sharp}$ its dual vector field (precisely, see Section 2). Then we have the following well-known theorem.

THEOREM A ([6]). Let $\left(M, g_{M}, \mathscr{F}\right)$ be a closed, connected Riemannian manifold with a Riemannian foliation $\mathscr{F}$ of a nonzero constant transversal scalar curvature $\sigma^{Q}$. If $M$ admits a transversal nonisometric conformal field $Y$ satisfying one of the following conditions:

(1) $Y=\nabla h$ for any basic function $h$, or

(2) $L_{Y} \operatorname{Ric}^{Q}=\mu g_{Q}$ for some basic function $\mu$, or

(3) $\operatorname{Ric}^{Q}\left(\nabla f_{Y}\right)=\frac{\sigma^{Q}}{q} \nabla f_{Y}, g_{Q}\left(\kappa_{B}^{\sharp}, \nabla f_{Y}\right)=0$ and $g_{Q}\left(A_{\kappa_{B}^{\sharp}} \nabla f_{Y}, \nabla f_{Y}\right) \leq 0$, then $\mathscr{F}$ is transversally isometric to $\left(S^{q}(1 / c), G\right)$.

Now, we recall two tensor fields $E^{Q}$ and $Z^{Q}$ ([3], [5]) by

$$
\begin{aligned}
& E^{Q}(Y)=\operatorname{Ric}^{Q}(Y)-\frac{\sigma^{Q}}{q} Y, \quad Y \in T \mathscr{F}^{\perp}, \\
& Z^{Q}(X, Y)=R^{Q}(X, Y)-R_{\sigma}^{Q}(X, Y),
\end{aligned}
$$

where $R_{\sigma}^{Q}(X, Y) s=\frac{\sigma^{Q}}{q(q-1)}\left\{g_{Q}(\pi(Y), s) \pi(X)-g_{Q}(\pi(X), s) \pi(Y)\right\}$ for any vector field $X, Y \in T M$ and $s \in \Gamma Q$. Trivially, if $E^{Q}=0$ (resp. $Z^{Q}=0$ ), then the foliation is transversally Einsteinian (resp. transversally constant sectional curvature). The tensor $Z^{Q}$ is called as the transversal concircular curvature tensor, which is a generalization of the concircular curvature tensor on a 
Riemannian manifold. In an ordinary manifold, the concircular curvature tensor is invariant under a concircular transformation which is a conformal transformation preserving geodesic circles [17]. Then we have the well-known theorem.

TheOREM B ([3]). Let $\left(M, g_{M}, \mathscr{F}\right)$ be as in Theorem A. If $M$ admits a transversal nonisometric conformal field $Y$ such that

$$
\int_{M} g_{Q}\left(E^{Q}\left(\nabla f_{Y}\right), \nabla f_{Y}\right) \geq 0
$$

then $\mathscr{F}$ is transversally isometric to $\left(S^{q}(1 / c), G\right)$.

REMARK 1. Since $\operatorname{Ric}^{\mathrm{Q}}\left(\nabla f_{Y}\right)=\frac{\sigma^{Q}}{q} \nabla f_{Y}$ implies $E^{Q}\left(\nabla f_{Y}\right)=0$, Theorem B is a generalization of Theorem A (3) when $\mathscr{F}$ is minimal.

TheOREM C ([4], [5]). Let $\left(M, g_{M}, \mathscr{F}\right)$ be as in Theorem A, and suppose that $\mathscr{F}$ is minimal. If $M$ admits a transversal nonisometric conformal field $Y$ such that

$$
\text { (i) } \quad L_{Y}\left|E^{Q}\right|^{2}=0([4])
$$

or

$$
\text { (ii) } L_{Y}\left|Z^{Q}\right|^{2}=0([5]) \text {, }
$$

then $\mathscr{F}$ is transversally isometric to $\left(S^{q}(1 / c), G\right)$.

REMARK 2. Theorem B and Theorem C have been proved in [18] for the point foliation, that is, for ordinary manifolds.

In this paper, we prove the following theorems.

THEOREM 1. Let $\left(M, g_{M}, \mathscr{F}\right)$ be as in Theorem A, and suppose that $\mathscr{F}$ is minimal. If $M$ admits a transversal nonisometric conformal field $Y$ such that

$$
L_{Y}\left|E^{Q}\right|^{2}=\text { const. } \quad \text { or } \quad L_{Y}\left|Z^{Q}\right|^{2}=\text { const. },
$$

then $\mathscr{F}$ is transversally isometric to $\left(S^{q}(1 / c), G\right)$.

REMARK 3. Theorem 1 is a generalization of Theorem C.

THEOREM 2. Let $\left(M, g_{M}, \mathscr{F}\right)$ be as in Theorem A, and suppose that $\mathscr{F}$ is minimal. If $M$ admits a transversal nonisometric conformal field $Y$ such that

$$
L_{Y} g_{Q}\left(L_{Y} E^{Q}, E^{Q}\right) \leq 0,
$$

then $\mathscr{F}$ is transversally isometric to $\left(S^{q}(1 / c), G\right)$. 
REMARK 4. Theorem 2 is a generalization of Theorem A (2) and (3) when $\mathscr{F}$ is minimal (cf. Remark 4.3). See also [19] for the ordinary manifold.

THEOREM 3. Let $\left(M, g_{M}, \mathscr{F}\right)$ be as in Theorem A. If $M$ admits a transversal conformal field $Y$ such that $Y=K+\nabla h$, where $K$ is a transversal Killing field and $h$ is a basic function, then $\mathscr{F}$ is transversally isometric to $\left(S^{q}(1 / c), G\right)$.

REMARK 5. Theorem 3 is a generalization of Theorem A (1).

\section{Preliminaries}

Let $\left(M, g_{M}, \mathscr{F}\right)$ be a $(p+q)$-dimensional Riemannian manifold with a foliation $\mathscr{F}$ of codimension $q$ and a bundle-like metric $g_{M}$ with respect to $\mathscr{F}$ [15]. Let $T M$ be the tangent bundle of $M, T \mathscr{F}$ its integrable subbundle given by $\mathscr{F}$, and $Q=T M / T \mathscr{F}$ the corresponding normal bundle. Then there exists an exact sequence of vector bundles

$$
0 \rightarrow T \mathscr{F} \rightarrow T M \underset{\sigma}{\stackrel{\pi}{\rightleftarrows}} Q \rightarrow 0,
$$

where $\pi: T M \rightarrow Q$ is a natural projection and $\sigma: Q \rightarrow T \mathscr{F}^{\perp}$ is a bundle map satisfying $\pi \circ \sigma=$ id. Let $g_{Q}$ be the holonomy invariant metric on $Q$ induced by $g_{M}$, that is, $L_{X} g_{Q}=0$ for any $X \in T \mathscr{F}$, where $L_{X}$ is the transversal Lie derivative, which is defined by $L_{X} s=\pi[X, \sigma(s)]$ for any $s \in \Gamma Q$. Let $\nabla$ be the transverse Levi-Civita connection in $Q$ [7]. The transversal curvature tensor $R^{Q}$ of $\nabla$ is defined by $R^{Q}(X, Y)=\left[\nabla_{X}, \nabla_{Y}\right]-\nabla_{[X, Y]}$ for any vector fields $X, Y \in \Gamma T M$. Let $\mathrm{Ric}^{Q}$ and $\sigma^{Q}$ be the transversal Ricci operator and the transversal scalar curvature of $\mathscr{F}$, respectively. The foliation $\mathscr{F}$ is said to be (transversally) Einsteinian if $\operatorname{Ric}^{Q}=\frac{1}{q} \sigma^{Q}$. id with constant transversal scalar curvature $\sigma^{Q}$. The mean curvature vector field $\tau$ is defined by

$$
\tau=\sum_{i=1}^{p} \pi\left(\nabla_{f_{i}}^{M} f_{i}\right)
$$

where $\left\{f_{i}\right\}(i=1, \ldots, p)$ is a local orthonormal frame field on $T \mathscr{F}$. The foliation $\mathscr{F}$ is said to be minimal if the mean curvature vector field $\tau$ vanishes. Let $\left\{e_{a}\right\}(a=1, \ldots, q)$ be a local orthonormal frame field on $Q$. For any $s \in \Gamma Q$, the transversal divergence $\operatorname{div}_{\nabla}(s)$ is given by

$$
\operatorname{div}_{\nabla}(s)=\sum_{a=1}^{q} g_{Q}\left(\nabla_{e_{a}} s, e_{a}\right) .
$$


For the later use, we recall the transversal divergence theorem [20] on a foliated Riemannian manifold.

THEOREM 1 ([20]). Let $\left(M, g_{M}, \mathscr{F}\right)$ be a closed, connected Riemannian manifold with a foliation $\mathscr{F}$ and a bundle-like metric $g_{M}$ with respect to $\mathscr{F}$. Then

$$
\int_{M} \operatorname{div}_{\nabla}(s)=\int_{M} g_{Q}(s, \tau)
$$

for all $s \in \Gamma Q$.

A differential form $\omega \in \Omega^{r}(M)$ is basic if $i(X) \omega=0$ and $i(X) d \omega=0$ for all $X \in T \mathscr{F}$, where $i(X)$ is the interior product. Let $\Omega_{B}^{r}(\mathscr{F})$ be the set of all basic r-forms on $M$. Then $\Omega^{*}(M)=\Omega_{B}^{*}(\mathscr{F}) \oplus \Omega_{B}^{*}(\mathscr{F})^{\perp}[1]$. Let $\kappa$ be the mean curvature form of $\mathscr{F}$, which is given by

$$
\kappa(s)=g_{Q}(\tau, s)
$$

for any $s \in Q$. Then the basic part $\kappa_{B}$ of the mean curvature form is closed, i.e., $d \kappa_{B}=0$ [1]. Let $d_{B}$ be the restriction of $d$ on $\Omega_{B}(\mathscr{F})$ and $\delta_{B}$ its formal adjoint operator of $d_{B}$ with respect to the global inner product $\langle\langle\cdot, \cdot\rangle\rangle$, which is given by

$$
\langle\langle\phi, \psi\rangle\rangle=\int_{M} \phi \wedge \bar{*} \psi \wedge \chi_{\mathscr{F}}
$$

for any basic $r$-forms $\phi$ and $\psi$, where $\bar{*}$ is the star operator on $\Omega_{B}^{*}(\mathscr{F})$ and $\chi_{\mathscr{F}}$ is the characteristic form of $\mathscr{F}$ [15]. The operator $\delta_{B}$ is given by

$$
\delta_{B} \phi=\left(\delta_{T}+i\left(\kappa_{B}^{\sharp}\right)\right) \phi, \quad \delta_{T} \phi=(-1)^{q(r+1)+1} \bar{*} d_{B} \bar{*} \phi .
$$

Note that the induced connection $\nabla$ on $\Omega_{B}^{*}(\mathscr{F})$ from the connection $\nabla$ on $Q$ and Riemannian connection $\nabla^{M}$ on $M$ extends the partial Bott connection, which satisfies $\nabla_{X} \omega=L_{X} \omega$ for any $X \in T \mathscr{F}$ [9]. Then the operator $\delta_{T}$ is given by

$$
\delta_{T} \phi=-\sum_{a=1}^{q} i\left(e_{a}\right) \nabla_{e_{a}} \phi .
$$

The basic Laplacian $\Delta_{B}$ acting on $\Omega_{B}^{*}(\mathscr{F})$ is defined by

$$
\Delta_{B}=d_{B} \delta_{B}+\delta_{B} d_{B}
$$

Then for any basic function $f$, we have

$$
\Delta_{B} f=\delta_{B} d_{B} f=-\sum_{a} \nabla_{e_{a}} \nabla_{e_{a}} f+\kappa_{B}^{\sharp}(f) .
$$


REMARK 6. Note that for any basic form $\omega$, the relation between $\delta_{B}$ and the ordinary operator $\delta$ is given by

$$
\delta \omega=\delta_{B} \omega+* \gamma(\omega)
$$

where $\gamma(\omega)= \pm \bar{*} \omega \wedge \varphi_{0} \quad$ and $\quad \varphi_{0}=d \chi_{\mathscr{F}}+\kappa \wedge \chi_{\mathscr{F}} \quad$ with $\quad \varphi_{0} \wedge \chi_{\mathscr{F}}=0 \quad$ [15]. If $\omega \in \Omega_{B}^{r}(r=0,1)$, then we easily have

$$
\gamma(\omega)=0
$$

which implies that

$$
\delta \omega=\delta_{B} \omega, \quad \Delta^{M} \omega=\Delta_{B} \omega,
$$

where $\Delta^{M}=d \delta+\delta d$ is the ordinary Laplacian.

For later use, we recall the generalized maximum principle for foliation ([6]).

TheOREM 2 ([6]). Let $\left(M, g_{M}, \mathscr{F}\right)$ be a closed, connected Riemannian manifold with a foliation $\mathscr{F}$ and a bundle-like metric $g_{M}$. For any basic function $f$, the condition $\left(\Delta_{B}-\kappa_{B}^{\sharp}\right) f \geq 0$ implies that $f$ is constant.

And we review some theorems for transversal nonisometric conformal field ([4]).

THEOREM 3 ([4]). Let $\left(M, g_{M}, \mathscr{F}\right)$ be a closed, connected Riemannian manifold with a foliation $\mathscr{F}$ of codimension $q$ and bundle-like metric $g_{M}$ such that $\delta_{B} \kappa_{B}=0$. Assume that the transversal scalar curvature $\sigma^{Q}$ is nonzero constant. Then for any transversal nonisometric conformal field $Y$ such that $L_{Y} g_{Q}=2 f_{Y} g_{Q}\left(f_{Y} \neq 0\right)$,

$$
\left(\Delta_{B}-\kappa_{B}^{\sharp}\right) f_{Y}=\frac{\sigma^{Q}}{q-1} f_{Y} \quad \text { and } \quad \int_{M} f_{Y}=0 .
$$

\section{Tensors $E^{Q}$ and $Z^{Q}$}

In this section, we give the properties of tensors $E^{Q}$ and $Z^{Q}$ on a Riemannian foliation. From (1) and (2), we have

$$
\sum_{a} Z^{Q}\left(s, e_{a}\right) e_{a}=E^{Q}(s)
$$

for any $s \in \Gamma Q$. Also, we have the following ([4], [5]). 


$$
\begin{aligned}
& \operatorname{tr}_{Q} E^{Q}=0, \quad \operatorname{div}_{\nabla}\left(E^{Q}\right)=\frac{q-2}{2 q} \nabla \sigma^{Q} \\
& \left|E^{Q}\right|^{2}=\left|\operatorname{Ric}^{Q}\right|^{2}-\frac{\left(\sigma^{Q}\right)^{2}}{q}, \quad\left|Z^{Q}\right|^{2}=\left|R^{Q}\right|^{2}-\frac{2\left(\sigma^{Q}\right)^{2}}{q(q-1)} \quad \text { if } q \geq 2 .
\end{aligned}
$$

Now, we recall the Lie derivatives of tensors along the transversal conformal field.

Lemma 1 ([3], [4], [5]). Let $Y$ be a transversal conformal field such that $L_{Y} g_{Q}=2 f_{Y} g_{Q} . \quad$ Then

$$
\begin{aligned}
& g_{Q}\left(\left(L_{Y} R^{Q}\right)\left(e_{a}, e_{b}\right) e_{c}, e_{d}\right)=\delta_{b}^{d} \nabla_{a} f_{c}-\delta_{b}^{c} \nabla_{a} f_{d}-\delta_{a}^{d} \nabla_{b} f_{c}+\delta_{a}^{c} \nabla_{b} f_{d}, \\
& \left(L_{Y} \operatorname{Ric}^{Q}\right)\left(e_{a}, e_{b}\right)=-(q-2) \nabla_{a} f_{b}+\left(\Delta_{B} f_{Y}-\kappa_{B}^{\sharp}\left(f_{Y}\right)\right) \delta_{a}^{b}, \\
& L_{Y} \sigma^{Q}=2(q-1)\left(\Delta_{B} f_{Y}-\kappa_{B}^{\sharp}\left(f_{Y}\right)\right)-2 f_{Y} \sigma^{Q}, \\
& \left(L_{Y} E^{Q}\right)\left(e_{a}, e_{b}\right)=-(q-2)\left\{\nabla_{a} f_{b}+\frac{1}{q}\left(\Delta_{B} f-\kappa_{B}^{\sharp}(f)\right) \delta_{a}^{b}\right\}, \\
& L_{Y}\left|E^{Q}\right|^{2}=-2(q-2) g_{Q}\left(\nabla \nabla f_{Y}, E^{Q}\right)-4 f_{Y}\left|E^{Q}\right|^{2}, \\
& L_{Y}\left|Z^{Q}\right|^{2}=-8 g_{Q}\left(\nabla \nabla f_{Y}, E^{Q}\right)-4 f_{Y}\left|Z^{Q}\right|^{2} .
\end{aligned}
$$

where $\nabla_{a}=\nabla_{e_{a}}$ and $f_{a}=\nabla_{a} f_{Y}$.

LeMma 2. If a transversal conformal field $Y$ satisfies $L_{Y} \operatorname{Ric}^{Q}=\mu g_{Q}$ for some basic function $\mu$, then

$$
L_{Y} E^{Q}=0 .
$$

Proof. Let $Y$ be the transversal conformal field such that $L_{Y} g_{Q}=2 f_{Y} g_{Q}$. From (3.4), we have

$$
-(q-2) \nabla_{a} f_{b}+\left(\Delta_{B} f_{Y}-\kappa_{B}^{\sharp}\left(f_{Y}\right)\right) \delta_{a}^{b}=\mu \delta_{a}^{b} .
$$

From (3) and (13), we have

$$
\mu=\frac{2(q-1)}{q}\left(\Delta_{B} f_{Y}-\kappa_{B}^{\sharp}\left(f_{Y}\right)\right) .
$$

From (13) and (14), we have

$$
-(q-2)\left\{\nabla_{a} f_{b}+\frac{1}{q}\left(\Delta_{B} f_{Y}-\kappa_{B}^{\sharp}\left(f_{Y}\right)\right) \delta_{a}^{b}\right\}=0 .
$$

Therefore, the proof follows from (10). 
Lemma 3. If $Y$ is a transversal conformal field, then

$$
L_{Y}\left|E^{Q}\right|^{2}=2 g_{Q}\left(L_{Y} E^{Q}, E^{Q}\right) .
$$

Proof. Let $\left\{e_{a}\right\}$ be a local orthonormal basis on $Q$ such that $\left(\nabla e_{a}\right)_{x}=0$ at a point $x$. Let $Y$ be the transversal conformal field $Y$ such that $L_{Y} g_{Q}=$ $2 f_{Y} g_{Q}$. Then at $x$, we have

$$
\begin{aligned}
L_{Y}\left|E^{Q}\right|^{2}= & \sum_{a} L_{Y} g_{Q}\left(E^{Q}\left(e_{a}\right), E^{Q}\left(E_{a}\right)\right) \\
= & \sum_{a}\left(L_{Y} g_{Q}\right)\left(E^{Q}\left(e_{a}\right), E^{Q}\left(e_{a}\right)\right)+2 \sum_{a} g_{Q}\left(\left(L_{Y} E^{Q}\right)\left(e_{a}\right), E^{Q}\left(e_{a}\right)\right) \\
& +2 \sum_{a} g_{Q}\left(E^{Q}\left(L_{Y} e_{a}\right), E^{Q}\left(e_{a}\right)\right) \\
= & 2 f_{Y}\left|E^{Q}\right|^{2}+2 g_{Q}\left(L_{Y} E^{Q}, E^{Q}\right)+2 \sum_{a} g_{Q}\left(E^{Q}\left(L_{Y} e_{a}\right), E^{Q}\left(e_{a}\right)\right) .
\end{aligned}
$$

Now, we calculate the last term in the above equation. That is,

$$
\begin{aligned}
& \sum_{a} g_{Q}\left(E^{Q}\left(L_{Y} e_{a}\right), E^{Q}\left(e_{a}\right)\right) \\
&=\sum_{a, b} g_{Q}\left(E^{Q_{(}}\left(L_{Y} e_{a}\right), e_{b}\right) g_{Q}\left(E^{Q}\left(e_{a}\right), e_{b}\right) \\
&= \sum_{a, b} g_{Q}\left(E^{Q}\left(e_{b}\right), L_{Y} e_{a}\right) g_{Q}\left(E^{Q}\left(e_{b}\right), e_{a}\right) \\
&= \frac{1}{2} \sum_{a, b} L_{Y}\left\{g_{Q}\left(E^{Q}\left(e_{b}\right), e_{a}\right) g_{Q}\left(E^{Q}\left(e_{b}\right), e_{a}\right)\right\}-2 f_{Y}\left|E^{Q}\right|^{2} \\
&-\sum_{a} g_{Q}\left(\left(L_{Y} E^{Q}\right)\left(e_{a}\right), E^{Q}\left(e_{a}\right)\right)-\sum_{a} g_{Q}\left(E^{Q}\left(L_{Y} e_{a}\right), E^{Q}\left(e_{a}\right)\right) .
\end{aligned}
$$

Hence we have

$$
\begin{aligned}
2 \sum_{a} g_{Q}\left(E^{Q}\left(L_{Y} e_{a}\right), E^{Q}\left(e_{a}\right)\right)= & \frac{1}{2} L_{Y}\left|E^{Q}\right|^{2}-2 f_{Y}\left|E^{Q}\right|^{2} \\
& -g_{Q}\left(L_{Y} E^{Q}, E^{Q}\right) .
\end{aligned}
$$

From (15) and (16), the proof is completed.

LeMma 4. Let $Y$ be a transversal conformal field such that $L_{Y} g_{Q}=2 f_{Y} g_{Q}$. Then 


$$
\begin{aligned}
& L_{Y}\left|Z^{Q}\right|^{2}=2 g_{Q}\left(L_{Y} Z^{Q}, Z^{Q}\right)-4 f_{Y}\left|Z^{Q}\right|^{2} \\
& (q-2) g_{Q}\left(L_{Y} Z^{Q}, Z^{Q}\right)=4 g_{Q}\left(L_{Y} E^{Q}, E^{Q}\right)+8 f_{Y}\left|E^{Q}\right|^{2} .
\end{aligned}
$$

Proof. Note that $g_{Q}\left(L_{Y} Z^{Q}, Z^{Q}\right)=-4 g_{Q}\left(\nabla \nabla f_{Y}, E^{Q}\right)$ [5]. So (17) follows from (12). For the proof of (18), from (11) and (12),

$$
4 L_{Y}\left|E^{Q}\right|^{2}=(q-2) L_{Y}\left|Z^{Q}\right|^{2}+4(q-2) f_{Y}\left|Z^{Q}\right|^{2}-16 f_{Y}\left|E^{Q}\right|^{2} .
$$

Hence from Lemma 3.3 and (17), the equation (18) is proved.

From (6) and Theorem C, we have the following.

Proposition 1. Let $\left(M, g_{M}, \mathscr{F}\right)$ be a closed, connected Riemannian manifold with a minimal foliation $\mathscr{F}$ of codimension $q \geq 2$ and a bundle-like metric $g_{M}$. Assume that the transversal scalar curvature is nonzero constant and either $\left|\operatorname{Ric}^{Q}\right|$ or $\left|R^{Q}\right|$ is constant. If $M$ admits a transversal nonisometric conformal field, then $\mathscr{F}$ is transversally isometric to $\left(S^{q}(1 / c), G\right)$.

Remark 7. For the ordinary manifold, Proposition 3.5 has been proved in [2] and [11], respectively.

\section{The proofs of Theorems}

First, we recall the integral formulas for the tensor $E^{Q}$ and $Z^{Q}$.

Proposition 2 ([3], [5]). Let $\left(M, g_{M}, \mathscr{F}\right)$ be a closed, connected Riemannian manifold with a foliation $\mathscr{F}$ of codimension $q$ and a bundle-like metric $g_{M}$ with respect to $\mathscr{F}$. Assume that the transversal scalar curvature $\sigma^{Q}$ is nonzero constant. Then for any transversal nonisometric conformal field $Y$ such that $L_{Y} g_{Q}=2 f_{Y} g_{Q}\left(f_{Y} \neq 0\right)$, we have

$$
\begin{aligned}
2(q-2) \int_{M} g_{Q}\left(E^{Q}\left(\nabla f_{Y}\right), \nabla f_{Y}\right)= & \int_{M}\left\{4 f_{Y}^{2}\left|E^{Q}\right|^{2}+f_{Y} L_{Y}\left|E^{Q}\right|^{2}\right\} \\
& +2(q-2) \int_{M} g_{Q}\left(E^{Q}\left(f_{Y} \nabla f_{Y}\right), \kappa_{B}^{\sharp}\right)
\end{aligned}
$$

and

$$
\begin{aligned}
\int_{M} g_{Q}\left(E^{Q}\left(\nabla f_{Y}\right), \nabla f_{Y}\right)= & \frac{1}{2} \int_{M}\left\{f_{Y}^{2}\left|Z^{Q}\right|^{2}+\frac{1}{4} f_{Y} L_{Y}\left|Z^{Q}\right|^{2}\right\} \\
& \int_{M} g_{Q}\left(\operatorname{Ric}^{Q}\left(f_{Y} \nabla f_{Y}\right), \kappa_{B}^{\sharp}\right)
\end{aligned}
$$


Proof of Theorem 1. Let $Y$ be the transversal nonisometric conformal field such that $L_{Y} g_{Q}=2 f_{Y} g_{Q}$. From Theorem 2.3, we have

$$
\int_{M} f_{Y}=0
$$

Assume that $\mathscr{F}$ is minimal. Since $L_{Y}\left|E^{Q}\right|^{2}=$ const or $L_{Y}\left|Z^{Q}\right|^{2}=$ const, from (19) and Proposition 4.1, we have

$$
2(q-2) \int_{M} g_{Q}\left(E^{Q}\left(\nabla f_{Y}\right), \nabla f_{Y}\right)=4 \int_{M} f_{Y}^{2}\left|E^{Q}\right|^{2}
$$

or

$$
\int_{M} g_{Q}\left(E^{Q}\left(\nabla f_{Y}\right), \nabla f_{Y}\right)=\frac{1}{2} \int_{M} f_{Y}^{2}\left|Z^{Q}\right|^{2},
$$

respectively. Hence from Theorem B, the proof is completed.

LeMma 5. Let $Y$ be a transversal conformal field such that $L_{Y} g_{Q}=2 f_{Y} g_{Q}$. Then for any basic function $h$,

$$
\int_{M} h f_{Y}=-\frac{1}{q} \int_{M} L_{Y} h+\frac{1}{q} \int_{M} \operatorname{div}_{\nabla}(h Y) .
$$

Proof. Let $\omega=Y^{b}$ be the dual basic 1-form of the transversal conformal form $Y$. Then

$$
\int_{M} h\left(\delta_{B} \omega\right)=\int_{M} g_{Q}\left(\omega, d_{B} h\right)=\int_{M} i(Y) d_{B} h=\int_{M} L_{Y} h .
$$

Since $\delta_{B}=\delta_{T}+i\left(\kappa_{B}^{\sharp}\right)$ and $\delta_{T} \omega=-\operatorname{div}_{\nabla}(Y)=-q f_{Y}$, we have

$$
\begin{aligned}
q \int_{M} h f_{Y} & =-\int_{M} h\left(\delta_{T} \omega\right) \\
& =-\int_{M} h\left(\delta_{B} \omega\right)+\int_{M} h i\left(\kappa_{B}^{\sharp}\right) \omega \\
& =-\int_{M} L_{Y} h+\int_{M} g_{Q}\left(h Y, \kappa_{B}^{\sharp}\right) \\
& =-\int_{M} L_{Y} h+\int_{M} \operatorname{div}_{\nabla}(h Y) .
\end{aligned}
$$

Last equality in above follows from the transversal divergence theorem (Theorem 2.1). Therefore, the proof is completed. 
Proof of Theorem 2. Let $Y$ be a transversal nonisometric conformal field, i.e., $L_{Y} g_{Q}=2 f_{Y} g_{Q}$. From (4), Lemma 3.4 and Proposition 4.1, if we put $h=g_{Q}\left(L_{Y} E^{Q}, E^{Q}\right)$, then from Lemma 4.2, we have

$$
\begin{aligned}
(q-2) & \int_{M} g_{Q}\left(E\left(\nabla f_{Y}\right), \nabla f_{Y}\right) \\
= & 2 \int_{M} f_{Y}^{2}\left|E^{Q}\right|^{2}+\int_{M} h f_{Y}+(q-2) \int_{M} g_{Q}\left(E\left(f_{Y} \nabla f_{Y}\right), \kappa_{B}^{\sharp}\right) \\
= & 2 \int_{M} f_{Y}^{2}\left|E^{Q}\right|^{2}-\frac{1}{q} \int_{M} L_{Y} h+\frac{1}{q} \int_{M} g_{Q}\left(h Y, \kappa_{B}^{\sharp}\right) \\
& +(q-2) \int_{M} g_{Q}\left(E^{Q}\left(f_{Y} \nabla f_{Y}\right), \kappa_{B}^{\sharp}\right) .
\end{aligned}
$$

Since $\mathscr{F}$ is minimal, we have

$$
(q-2) \int_{M} g_{Q}\left(E^{Q}\left(\nabla f_{Y}\right), \nabla f_{Y}\right)=2 \int_{M} f_{Y}^{2}\left|E^{Q}\right|^{2}-\frac{1}{q} \int_{M} L_{Y} g_{Q}\left(L_{Y} E^{Q}, E^{Q}\right) .
$$

Hence by the condition $L_{Y} g_{Q}\left(L_{Y} E^{Q}, E^{Q}\right) \leq 0$, we have

$$
\int_{M} g_{Q}\left(E^{Q}\left(\nabla f_{Y}\right), \nabla f_{Y}\right) \geq 0 \text {. }
$$

From Theorem B, the proof of Theorem 2 is completed.

REMARK 8. Let $\mathscr{F}$ be minimal. Then the following holds.

(1) From Lemma 3.2, Theorem 2 yields Theorem A (2).

(2) Theorem 2 is also a generalization of Theorem A (3). In fact, assume that $\operatorname{Ric}^{Q}\left(\nabla f_{Y}\right)=\frac{\sigma^{Q}}{q} \nabla f_{Y}$, that is, $E^{Q}\left(\nabla f_{Y}\right)=0 . \quad$ By differentiation, we have

$$
\left(\nabla_{e_{a}} E^{Q}\right)\left(\nabla f_{Y}\right)+E^{Q}\left(\nabla_{a} \nabla f_{Y}\right)=0 .
$$

From (20), we have

$$
\begin{aligned}
0 & =\sum_{a} g_{Q}\left(\left(\nabla_{e_{a}} E^{Q}\right)\left(\nabla f_{Y}\right)+E^{Q}\left(\nabla_{a} \nabla f_{Y}\right), e_{a}\right) \\
& =g_{Q}\left(\nabla f_{Y}, \operatorname{div}_{\nabla}\left(E^{Q}\right)\right)+\sum_{a} g_{Q}\left(E^{Q}\left(\nabla_{a} \nabla f_{Y}\right), e_{a}\right) \\
& =\sum_{a} g_{Q}\left(\nabla_{a} \nabla f_{Y}, E^{Q}\left(e_{a}\right)\right) .
\end{aligned}
$$

From (5), $\operatorname{div}_{\nabla} E^{Q}=0$ and so the last equality in the above follows. Hence from (10) and (21), we have 


$$
\begin{aligned}
g_{Q}\left(L_{Y} E^{Q}, E^{Q}\right)= & \sum_{a} g_{Q}\left(\left(L_{Y} E^{Q}\right)\left(e_{a}\right), E^{Q}\left(e_{a}\right)\right) \\
= & -(q-2) \sum_{a} g_{Q}\left(\nabla \nabla f_{Y}, E^{Q}\left(e_{a}\right)\right) \\
& -\frac{q-2}{q}\left(\Delta_{B} f_{Y}\right) \sum_{a} g_{Q}\left(e_{a}, E^{Q}\left(e_{a}\right)\right) \\
= & -(q-2) \sum_{a} g_{Q}\left(\nabla_{a} \nabla f_{Y}, E^{Q}\left(e_{a}\right)\right)-\frac{q-2}{q}\left(\Delta_{B} f_{Y}\right) \operatorname{tr}_{Q} E^{Q} \\
= & 0 .
\end{aligned}
$$

The last equality follows from $\operatorname{tr}_{Q} E^{Q}=0$. Hence the conditions of Theorem A (3) implies that $g_{Q}\left(L_{Y} E^{Q}, E^{Q}\right)=0$. That is, by Theorem 2, $\mathscr{F}$ is transversally isometric to the sphere.

Proof of Theorem 3. Let $Y$ be a transversal conformal field such that $L_{Y} g_{Q}=2 f_{Y} g_{Q}$ and $Y=K+\nabla h$, where $K$ is a transversal Killing field and $h$ is a basic function. Then

$$
g_{Q}\left(\nabla_{X} Y, Z\right)+g_{Q}\left(\nabla_{Z} Y, X\right)=2 f_{Y} g_{Q}(X, Z)
$$

for any normal vector field $X, Z \in \Gamma Q$. On the other hand, since the transversal scalar curvature $\sigma^{Q}$ is constant, from Theorem 2.4, we have

$$
\left(\Delta_{B}-\kappa_{B}^{\sharp}\right) f_{Y}=\frac{\sigma^{Q}}{q-1} f_{Y} .
$$

Since $Y=K+\nabla h$, we have $L_{Y} g_{Q}=L_{\nabla h} g_{Q}=2 f_{Y} g_{Q}$. That is,

$$
g_{Q}\left(\nabla_{X} \nabla h, Z\right)+g_{Q}\left(\nabla_{Z} \nabla h, X\right)=2 f_{Y} g_{Q}(X, Z) .
$$

On the other hand, $(\nabla \nabla h)(X, Z)=g_{Q}\left(\nabla_{X} \nabla h, Z\right)$ is symmetric. Therefore, from (23)

$$
(\nabla \nabla h)(X, Z)=f_{Y} g_{Q}(X, Z) .
$$

Hence from (3) and (24), we have

$$
\left(\Delta_{B}-\kappa_{B}^{\sharp}\right) h=-q f_{Y} .
$$

From (22) and (25), we get

$$
\left(\Delta_{B}-\kappa_{B}^{\sharp}\right)\left(f_{Y}+\frac{\sigma^{Q}}{q(q-1)} h\right)=0 .
$$


By the generalized maximum principle (Theorem 2.3), we have

$$
f_{Y}+\frac{\sigma^{Q}}{q(q-1)} h=\mathrm{const},
$$

which implies

$$
\nabla \nabla f_{Y}+\frac{\sigma^{Q}}{q(q-1)} \nabla \nabla h=0 .
$$

From (24) and (26), we have

$$
\nabla \nabla f_{Y}=-\frac{\sigma^{Q}}{q(q-1)} f_{Y} .
$$

By the generalized Obata theorem [6], $\mathscr{F}$ is transversally isometric to $\left(S^{q}(1 / c), G\right)$, where $c^{2}=\frac{\sigma^{Q}}{q(q-1)}$.

REMARK 9. Theorem 3 is a generalization of Theorem A (1).

\section{Acknowledgement}

The authors would like to thank the referee for his or her kind comments to improve this article.

\section{References}

[1] J. A. Alvarez López, The basic component of the mean curvature of Riemannian foliations, Ann. Global Anal. Geom. 10 (1992), 179-194.

[2] C. C. Hsiung, On the group of conformal transformations of a compact Riemannian manifold, Proc. Nat. Acad. Sci. U.S.A. 56 (1965), 1509-1513.

[3] S. D. Jung, Riemannian foliations admitting transversal conformal fields II, Geom. Dedicata 175 (2015), 257-266.

[4] M. J. Jung and S. D. Jung, Riemannian foliations admitting transversal conformal fields, Geom. Dedicata 133 (2008), 155-168.

[5] S. D. Jung and K. R. Lee, The properties of Riemannian foliations admitting transversal conformal fields, Bull. Korean Math. Soc. 55 (2018), 1273-1283.

[6] S. D. Jung, K. R. Lee and K. Richardson, Generalized Obata theorem and its applications on foliations, J. Math. Anal. Appl. 376 (2011), 129-135.

[7] F. W. Kamber and Ph. Tondeur, Harmonic foliations, Proc. National Science Foundation Conference on Harmonic Maps, Tulane, Dec. 1980, Lecture Notes in Math. 949, SpringerVerlag, New-York, 1982, 87-121.

[8] F. W. Kamber and $\mathrm{Ph}$. Tondeur, Infinitesimal automorphisms and second variation of the energy for harmonic foliations, Tohoku Math. J. 34 (1982), 525-538.

[9] F. W. Kamber and Ph. Tondeur, De Rham-Hodge theory for Riemannian foliations, Math. Ann. 277 (1987), 415-431. 
[10] J. Lee and K. Richardson, Lichnerowicz and Obata theorems for foliations, Pacific J. Math. 206 (2002), 339-357.

[11] A. Lichnerowicz, Sur les transformations conformes d'une variété riemannienne compacte, C. R. Acad. Sci. Paris 259 (1964), 697-700.

[12] H. K. Pak and J. H. Park, A note on generalized Lichnerowica-Obata theorems for Riemannian foliations, Bull. Korean Math. Soc. 48 (2011), 769-777.

[13] J. S. Pak and S. Yorozu, Transverse fields on foliated Riemannian manifolds, J. Korean Math. Soc. 25 (1988), 83-92.

[14] J. H. Park and S. Yorozu, Transversal conformal fields of foliations, Nihonkai Math. J. 4 (1993), 73-85.

[15] Ph. Tondeur, Geometry of foliations, Birkhäuser-Verlag, Basel; Boston: Berlin, 1997.

[16] Ph. Tondeur and G. Toth, On transversal infinitesimal automorphisms for harmonic foliations, Geom. Dedicata 24 (1987), 229-236.

[17] K. Yano, Concircular geometry I, Concircular transformations, Proc. Imp. Acad. Tokyo 16 (1940), 195-200.

[18] K. Yano, On Riemannian manifolds with constant scalar curvature admitting a conformal transformation group, Proc. Nat. Acad. Sci. U.S. 55 (1966), 472-476.

[19] K. Yano and S. Sawaki, Riemannioan manifolds admitting a conformal transformation group, J. Differential Geometry 2 (1968), 161-184.

[20] S. Yorozu and T. Tanemura, Green's theorem on a foliated Riemannian manifold and its applications, Acta Math. Hungar. 56 (1990), 239-245.

\author{
Woo Cheol Kim \\ Department of Mathematics \\ Jeju National University \\ Jeju 63243 \\ Republic of Korea \\ E-mail: curi1981@naver.com \\ Seoung Dal Jung \\ Department of Mathematics \\ Jeju National University \\ Jeju 63243 \\ Republic of Korea \\ E-mail: sdjung@jejunu.ac.kr
}

